\title{
Qualidade da dieta entre consumidores e não consumidores de carnes vermelhas e processadas: estudo ISA-Capital
}

\author{
Diet quality of consumers and non-consumers \\ of red and processed meats:
}

ISA-Capital Study

\author{
Camilla Fabiana Catto de FREITAS 1 \\ Aline Mendes PERALTA \\ Aline Martins CARVALHO ${ }^{1}$ \\ Antonio Augusto Ferreira CARIOCA ${ }^{1}$ \\ Regina Mara FISBERG ${ }^{1}$ \\ Dirce Maria Lobo MARCHIONI ${ }^{1}$
}

\section{R E S U M O}

\section{Objetivo}

Avaliar a qualidade da dieta entre consumidores e não consumidores de carnes vermelhas e processadas em residentes do município de São Paulo.

\section{Métodos}

Foram utilizados dados do estudo transversal do Inquérito de Saúde de São Paulo - 2008, com informações de 726 indivíduos que possuíam todas as informações dos inquéritos dietéticos, sendo 195 adolescentes, 272 adultos e 259 idosos. Os dados de consumo alimentar foram obtidos por dois recordatórios alimentares de 24 horas, aplicados em dias não consecutivos, incluindo final de semana e todas as estações do ano, e um questionário de frequência alimentar para estimar a frequência do consumo de carnes vermelhas e processadas. A ingestão alimentar habitual foi estimada pelo Multiple Source Method. O Índice de Qualidade da Dieta Revisado foi calculado a partir da ingestão habitual dos participantes. As recomendações da Organização Mundial de Saúde foram utilizadas para estimar a participação percentual dos macronutrientes no valor energético total.

\footnotetext{
1 Universidade de São Paulo, Faculdade de Saúde Pública, Departamento de Nutrição. Av. Dr. Arnaldo, 715, 01246-904, São Paulo, SP, Brasil. Correspondência para/Correspondence to: DML MARCHIONI. E-mail: <marchioni@usp.br>.

Apoio: Conselho Nacional de Desenvolvimento Científico e Tecnológico (481176/2008-0; 473778/2010-6) e Fundação de Amparo à Pesquisa do Estado de São Paulo (2012/08702-1).
} 


\section{Resultados}

O grupo de não consumidores apresentou maior pontuação média do índice $(p=0,006)$, do grupo das frutas integrais $(p=0,022)$, dos leites e derivados $(p<0,001)$ e menor pontuação média de gordura sólida, álcool e açúcar de adição $(p=0,039)$ e carnes, ovos e leguminosas $(p<0,001)$. Também se mostrou maior percentual de adequação de gordura no grupo de não consumidores de carnes vermelhas e processadas.

\section{Conclusão}

Sugere-se que não consumidores de carne vermelha e processada tenha melhor qualidade da dieta e maior adequação da contribuição energética dos lipídeos em comparação com consumidores desse alimento.

Palavras-chave: Carne. Dieta. Inquéritos sobre dietas. Produtos da carne.

\section{A B S T R A C T}

\section{Objective}

To evaluate the diet quality of consumers and non-consumers of red and processed meats in São Paulo city.

\section{Methods}

Data came from the Health Study of São Paulo - 2008, a cross-sectional study of 726 individuals who completed a dietary survey (195 adolescents, 272 adults, and 259 older adults). Diet was assessed by two 24-hour dietary recalls collected on two nonconsecutive days, including a weekend day and all seasons, and a food frequency questionnaire to estimate the frequency of red and processed meat consumption. We used the Multiple Source Method to estimate the usual food intake. The Diet Quality Index was calculated based on the usual food intake of the participants. The recommendations of the World Health Organization were used for estimating macronutrient intake adequacy.

\section{Results}

Non-consumers of red and processed meat presented higher score in: total score ( $p=0.006)$, group of fresh fruits $(p=0.022)$, and group of milk and dairy products $(p<0.001)$; and lower score in: group of fats and added sugar $(p=0.039)$ and group of meats, eggs, and beans $(p<0.001)$ than consumers of red and processed meats. Non-consumers of red and processed meats also presented higher fat intake adequacy.

\section{Conclusion}

Our results suggest that non-consumers of red and processed meats had higher diet quality and fat intake adequacy than consumers of red and processed meats.

Keywords: Meat. Diet. Diet surveys. Meat products.

\section{N T R O D U Ç Ã O}

O consumo excessivo de carnes vermelhas e processadas vem sendo apontado como um dos possíveis contribuintes para o aumento da incidência de doenças crônicas não-transmissíveis, como doenças cardiovasculares, diabetes, síndrome metabólica e diversos tipos de cânceres, principalmente câncer gástrico e colorretal ${ }^{1-9}$.

A ingestão excessiva de carnes vermelhas e processadas foi considerada elevada na cidade de São Paulo, em 2008, com aproximadamente $75,0 \%$ da população ingerindo mais que a recomendação da World Cancer Research Fund ${ }^{1}$ (WCRF) (máximo 500 gramas por semana) ${ }^{10}$. Segundo dados da "Pesquisa de Orçamento Familiar 2008-2009"11, a carne bovina apresentou alta prevalência de consumo alimentar (48,7\%), e as carnes processadas variaram de 3,1\% para salsicha a 5,3\% para linguiça. Entretanto, doenças crônicas não sofrem influência somente da ingestão alimentar proveniente do consumo de carnes vermelhas e processadas, mas do hábito alimentar, ou seja, da quantidade e qualidade geral da alimentação do indivíduo 1,10,12. Algumas ferramentas são utilizadas para realizar a classifi- 
cação da qualidade da dieta, como os índices alimentares, dentre os quais se pode citar o Índice de Qualidade da Dieta - Revisado (IQD-R), desenvolvido de acordo com o Guia Alimentar para a População Brasileira, sendo capaz de analisar simultaneamente diversos componentes da dieta e avaliar a qualidade da alimentação independentemente da quantidade de alimentos consumidos ${ }^{13}$.

Assim, este estudo tem o objetivo de avaliar a qualidade da dieta entre consumidores e não consumidores de carnes vermelhas e processadas em residentes do município de São Paulo.

\section{MÉ TO D OS}

Foram utilizados dados do estudo transversal Inquérito de Saúde de São Paulo (ISA-Capital) ${ }^{14}$. Trata-se de um inquérito domiciliar e telefônico, de base populacional, conduzido em 2008 e 2009 com o objetivo de analisar condições de saúde da população de residentes de domicílios particulares na área urbana do município de São Paulo. A amostragem foi probabilística complexa, por conglomerados, em dois estágios: setores censitários e domicílios (dados provenientes da Pesquisa Nacional por Amostra de Domicílios 2005, Instituto Brasileiro de Geografia e Estatística). Foram considerados seis domínios amostrais: adolescentes (12 a 19 anos), adultos (20 a 59 anos) e idosos (60 anos e mais), de ambos os sexos, totalizando 2691 participantes. Foram utilizadas informações de 726 indivíduos que possuíam todas as informações dos inquéritos dietéticos utilizados nessa análise, sendo 195 adolescentes, 272 adultos e 259 idosos.

No Inquérito de Saúde de São Paulo, os dados de consumo alimentar foram obtidos por dois Recordatórios Alimentares de 24h (R24h) por pessoa - o primeiro realizado no domicílio e o segundo por telefone -, sendo aplicados em dias não consecutivos, incluindo final de semana e todas as estações do ano, utilizando o Multiple Pass Methods ${ }^{15}$. Esse método contribui para que o indivíduo recorde-se dos alimentos e bebidas consumidos no dia anterior e relate-os de maneira detalhada, reduzindo erros de coleta ${ }^{16}$. Utilizou-se também um Questionário de Frequência Alimentar ${ }^{17,18}$ para estimar a frequência do consumo de carnes vermelhas e processadas. A ingestão alimentar habitual foi estimada pelo Multiple Source Method ${ }^{19}$, com o objetivo de atenuar a variância intrapessoal.

A determinação de consumidores de carne vermelha e processada foi realizada pela verificação do relato de consumo de pelo menos um tipo de carne vermelha ou processada (carne bovina, suína, carneiro, linguiça etc.) pelo Questionário de Frequência Alimentar ${ }^{17}$ ou R24h. O grupo de não consumidores não apresentou consumo de carnes ou apresentou apenas o consumo de carnes brancas, como de aves e peixes.

O Índice da Qualidade da Dieta - Revisado ${ }^{13}$ foi calculado a partir da ingestão habitual dos participantes. Esse indicador é constituído por uma escala de pontos de 0 a 100: quanto maior a pontuação, melhor é a qualidade da dieta. Tal método utiliza 12 componentes, com pontuação máxima de 5 pontos (frutas totais, frutas integrais, vegetais totais, vegetais verdes-escuros e alaranjandos e leguminosas, cereais totais, cereais integrais), com pontuação máxima de 10 pontos (leites e derivados, carnes, ovos e leguminosas, óleos, gordura saturada, sódio) e com pontuação máxima de 20 pontos (Gordura Sólida, Álcool e Açúcar de Adição [GordAA]).

As recomendações da World Health Organization $^{12}$ (WHO) foram utilizadas para estimar a participação percentual dos macronutrientes no valor energético total. Os intervalos das recomendações para ingestão de energia proveniente de macronutrientes foram utilizados para classificar os indivíduos em adequado, se o consumo percentual de energia fornecido pelo nutriente estivesse dentro do intervalo, ou inadequado, se estivesse fora do intervalo.

Na aferição do peso corporal para cálculo das recomendações da WHO, adotaram-se os procedimentos descritos pela mesma ${ }^{20}$. 
Para avaliação da qualidade da dieta entre os grupos de consumidores ou não de carnes vermelhas e processadas foram utilizados os testes t Student, avaliando anteriormente a normalidade e a igualdade de variância e o teste exato de Fisher. O software estatístico utilizado para as análises foi o Stata (Stata Corp., College Station, Texas, Estados Unidos) versão 12 e os valores de $p<0,05$ foram considerados significativos.

O estudo foi aprovado pelo Comitê de Ética e Pesquisa da Faculdade de Saúde Pública da Universidade de São Paulo (Protocolo n² 2001), e todos os participantes assinaram o Termo de Consentimento Livre e Esclarecido.

\section{RES U L T A D OS}

Foram encontrados 715 consumidores de carnes vermelhas e processadas e 11 não consumidores $(1,54 \%$ de prevalência de não consumidores).

As carnes processadas consideradas para as análises foram linguiça, salsicha e hambúrguer, que mais frequentemente substituem carnes frescas nas refeições diárias, sendo 78,8, 67,0 e $54,5 \%$ os percentuais de indivíduos consumidores que consumiram tais carnes, respectivamente.

Não houve diferenças quanto à faixa etária, renda, escolaridade e raça entre os grupos de consumidores e não consumidores (Tabela 1). A maioria tinha $\geq 20$ anos $(73,2 \%)$ e era do sexo feminino (58,3\%).

A dieta dos dois grupos foi semelhante na maioria dos componentes, entretanto o grupo de não consumidores apresentou maior média da pontuação geral do IQD-R $(p=0,006)$, do grupo das frutas integrais $(p=0,022)$, dos leites e derivados $(p<0,001)$ e menores pontuações do GordAA $(p=0,039)$, e carnes, ovos e leguminosas $(p<0,001)$ (Tabela 2).

A inadequação da contribuição energética dos macronutrientes foi alta, independentemente do grupo, tendo percentuais acima de $70 \%$ em todos os nutrientes, destacando-se para o consumo acima do recomendado de proteínas em

Tabela 1. Caracterização das amostras de acordo com o relato de consumo ou não de carnes vermelhas e processadas em população da cidade de São Paulo (SP), 2008.

\begin{tabular}{|c|c|c|c|c|c|c|c|}
\hline \multirow{2}{*}{ Variáveis } & \multicolumn{2}{|c|}{ Não consumidores } & \multicolumn{2}{|c|}{ Consumidores } & \multicolumn{2}{|c|}{ Total } & \multirow{2}{*}{$p^{*}$} \\
\hline & $\mathrm{n}$ & $\%$ & $n$ & $\%$ & $n$ & $\%$ & \\
\hline Faixa Etária & & & & & & & 0,177 \\
\hline Adolescentes (<20 anos) & 1 & 9,1 & 194 & 27,1 & 195 & 26,8 & \\
\hline Adultos ( $\geq 20$ e $<60$ anos) & 3 & 27,3 & 269 & 37,6 & 272 & 37,5 & \\
\hline Idosos ( $\geq 60$ anos) & 7 & 63,6 & 252 & 35,3 & 259 & 35,7 & \\
\hline Renda familiar & & & & & & & 0,365 \\
\hline Até um salário mínimo & 7 & 63,6 & 336 & 47,0 & 343 & 47,3 & \\
\hline Acima de um salário mínimo & 4 & 36,4 & 379 & 53,0 & 383 & 52,7 & \\
\hline Sexo & & & & & & & - \\
\hline Masculino & 0 & 0,0 & 303 & 42,4 & 303 & 41,7 & \\
\hline Feminino & 11 & 100,0 & 412 & 57,6 & 423 & 58,3 & \\
\hline Escolaridade & & & & & & & 1,000 \\
\hline Até 9 anos & 6 & 60,0 & 437 & 61,5 & 443 & 61,4 & \\
\hline 10 anos ou mais & 4 & 40,0 & 274 & 38,5 & 278 & 38,6 & \\
\hline Raça (autodeclarada) & & & & & & & 0,171 \\
\hline Branco & 5 & 45,4 & 421 & 59,0 & 426 & 58,7 & \\
\hline Não-branco ${ }^{1}$ & 6 & 54,6 & 294 & 41,0 & 300 & 41,3 & \\
\hline
\end{tabular}

Nota: ${ }^{*}$ Utilizou-se teste exato de Fisher; ${ }^{\mathbf{1}}$ Negros, pardos e indígenas. 
praticamente todos os indivíduos. Também houve associação entre a adequação da ingestão de lipídeos e os grupos, sendo que o grupo de não consumidores de carne vermelha e processada apresentou maior percentual de indivíduos com ingestão adequada de lipídeos em comparação ao grupo de consumidores de carnes vermelhas e processadas (Tabela 3).

Tabela 2. Valores médios de pontuação do Índice de Qualidade da Dieta - Revisado (IQD-R) e valores ingeridos (em kcal ou mg por $1.000 \mathrm{kcal}$ e percentual) dos componentes desse índice, de acordo com o relato de consumo ou não de carnes vermelhas e processadas em população da cidade de São Paulo (SP), 2008.

\begin{tabular}{|c|c|c|c|c|c|c|c|}
\hline \multirow{3}{*}{$\begin{array}{l}\text { Variáveis } \\
\text { IQDR }^{1}\end{array}$} & \multicolumn{3}{|c|}{ Não consumidores } & \multicolumn{3}{|c|}{ Consumidores } & \multirow{3}{*}{$\begin{array}{c}p^{*} \\
0,006\end{array}$} \\
\hline & \multirow{2}{*}{$\frac{M}{68,7}$} & \multirow{2}{*}{$\begin{array}{l}\mathrm{DP} \\
7,9\end{array}$} & IC95\% & \multirow{2}{*}{$\frac{M}{62,7}$} & \multirow{2}{*}{$\frac{\mathrm{DP}}{7,1}$} & IC95\% & \\
\hline & & & $63,4-74,0$ & & & $62,1-63,2$ & \\
\hline Frutas totais ${ }^{2}$ & 63,3 & 52,4 & $28,0-98,5$ & 49,5 & 35,0 & $46,9-52,0$ & 0,198 \\
\hline Frutas integrais ${ }^{3}$ & 64,4 & 53,2 & $28,6-100,1$ & 40,4 & 33,9 & $38,0-42,9$ & 0,022 \\
\hline Vegetais totais & 30,2 & 11,2 & $22,7-37,8$ & 34,8 & 14,0 & $33,8-35,8$ & 0,287 \\
\hline Vegetais verde alaranjados ${ }^{4}$ & 19,7 & 6,5 & $15,4-24,0$ & 22,5 & 9,0 & $21,8-23,1$ & 0,313 \\
\hline Leites e derivados ${ }^{5}$ & 158,0 & 64,5 & $114,7-201,4$ & 96,5 & 46,0 & $93,0-99,8$ & $<0,001$ \\
\hline Gordura Saturada (\%) & 9,9 & 1,9 & $8,5-11,2$ & 10,2 & 1,7 & $10,0-10,3$ & 0,556 \\
\hline GordAA ${ }^{6}(\%)$ & 21,0 & 5,5 & $17,4-24,8$ & 24,7 & 5,7 & $24,3-25,1$ & 0,039 \\
\hline Óleos ${ }^{7}$ & 71,3 & 19,4 & $58,3-84,3$ & 74,7 & 20,1 & $73,2-76,2$ & 0,578 \\
\hline Sódio & 1730,8 & 198,0 & $1597,8-1863,9$ & 1809,0 & 265,0 & $1789,6-1828,5$ & 0,330 \\
\hline Cereais totais $^{8}$ & 369,0 & 66,5 & $324,4-413,7$ & 358,3 & 66,4 & $353,5-363,2$ & 0,597 \\
\hline Cereais integrais & 26,5 & 34,5 & $3,4-49,7$ & 17,0 & 19,3 & $15,6-18,5$ & 0,112 \\
\hline Carnes, ovos e leguminosas & 128,9 & 30,4 & $108,4-149,3$ & 155,9 & 24,0 & $154,1-157,6$ & $<0,001$ \\
\hline
\end{tabular}

Nota: Valor de $p$ em negrito indica diferença significativa. * Utilizou-se teste $t$ Student; ${ }^{1}$ IQDR: Pontuação de 0 a $100 ;{ }^{2}$ Frutas totais: incluído frutas e sucos de frutas naturais; ${ }^{3}$ Frutas integrais: excluído sucos de frutas; ${ }^{4}$ Vegetais verdes-escuros, alaranjados e leguminosas: incluído leguminosas apenas depois que a pontuação máxima de carnes, ovos e leguminosas for atingida; 5 Leites e derivados: incluído leite e derivados e bebidas à base de soja; ${ }^{6}$ GordAA: calorias provenientes da Gordura Sólida, Álcool e Açúcar de Adição; ${ }^{7}$ Óleos: incluído as gorduras mono e poli-insaturadas, óleos das oleaginosas e gordura de peixe; ${ }^{8} \mathrm{C}$ ereais totais: representa o grupo dos cereais, raízes e tubérculos.

IC95\%: Intervalo de Confiança de 95\%; M: Média; DP: Desvio-Padrão.

Tabela 3. Valores percentuais de adequação de ingestão de macronutrientes de acordo com o relato de consumo ou não de carnes vermelhas e processadas em população da cidade de São Paulo (SP), 2008.

\begin{tabular}{|c|c|c|c|}
\hline \multirow{2}{*}{ Variáveis } & Não consumidores & Consumidores & \multirow{2}{*}{$p^{*}$} \\
\hline & $\%$ & $\%$ & \\
\hline \multicolumn{4}{|l|}{ Adequação da ingestão de carboidratos ${ }^{1}$} \\
\hline Adequado & 9,1 & 0,4 & \multirow{2}{*}{0,059} \\
\hline Inadequado (abaixo do recomendado) & 90,9 & 99,6 & \\
\hline \multicolumn{4}{|l|}{ Adequação da ingestão de proteínas² } \\
\hline Adequado & 0,0 & 0,7 & \multirow{2}{*}{0,926} \\
\hline Inadequado (acima do recomendado) & 100,0 & 99,3 & \\
\hline \multicolumn{4}{|l|}{ Adequação da ingestão de lipídeos ${ }^{3}$} \\
\hline Adequado & 27,3 & 7,1 & \multirow{2}{*}{0,042} \\
\hline Inadequado (acima do recomendado) & 72,7 & 92,9 & \\
\hline
\end{tabular}

Nota: *Utilizou-se teste exato de Fischer. ${ }^{1}$ Percentual de indivíduos com ingestão de carboidratos adequada ou inadequada, de acordo com a recomendação da World Health Organization ${ }^{12}$; ${ }^{2}$ Percentual de indivíduos com ingestão de proteínas adequada ou inadequada, de acordo com a recomendação da World Health Organization ${ }^{12}$; ${ }^{3}$ Percentual de indivíduos com ingestão de lipídeos adequada ou inadequada, de acordo com a recomendação da World Health Organization ${ }^{12}$. 


\section{I S C U S S Ã O}

O presente artigo analisou a qualidade da dieta de consumidores e não consumidores de carnes vermelhas e processadas em um estudo de base populacional representativo da cidade de São Paulo. Sugere-se que o grupo de não consumidores apresentou melhor qualidade da dieta, principalmente no que se relaciona aos componentes: frutas integrais, leites e derivados, gordura sólida, álcool, açúcar de adição, carnes, ovos e leguminosas.

O consumo de carnes proporciona benefícios nutricionais quando realizado em quantidades adequadas, segundo recomendações estabelecidas por organizações de saúde ${ }^{1,21}$. Porém, o consumo acima dos valores recomendados, principalmente o consumo de carnes vermelhas e processadas, pode ser fator de risco para doenças crônicas não transmissíveis, pois elas podem apresentar altas concentrações de gordura saturada, compostos cancerígenos como aminas heterocíclicas e hidrocarbonetos policíclicos aromáticos, que são formados no preparo das carnes, além da adição de conservantes como sódio, nitritos e nitratos ${ }^{1}$.

Dentre as diferenças da ingestão alimentar dos grupos estudados, observou-se que os consumidores de carne vermelha e processada tiveram uma menor ingestão de frutas. Outro estudo transversal representativo na Irlanda entre 1997-199922 mostrou dados semelhantes ao encontrado: consumidores de carnes processadas apresentaram ingestão menor de pão integral, legumes, frutas e peixe. Já Kappeler et al. ${ }^{23}$, com dados do Third National Health and Nutrition Examination Survey (NHANES III) (1986-2010), encontraram que consumidores de carnes vermelhas comeram menos frutas e mais vegetais, além de serem mais jovens e menos ativos em relação a outro grupo de indivíduos que consumiam menos carne vermelha.

Outro componente do IQD-R com diferença de ingestão entre os grupos foi o grupo dos leites e derivados. Camilleri et al. ${ }^{24}$, com dados de 1912 adultos do French Individual and National Consumption Survey 2 (2006-2007), comparou a ingestão de proteínas de origem animal e de origem vegetal e notou que, em comparação com as mulheres, os homens consumiram mais proteína de carne vermelha, aves, carnes processadas e cereais e menos proteínas de peixe, leite, iogurte. Fatos que sustentam o consumo maior de leite entre os não consumidores de carnes vermelhas e processadas, grupo constituído apenas por mulheres. Outros estudos observaram que mulheres apresentam maior aderência a um padrão prudente (caracterizado pelo consumo de frutas, legumes, verduras, pão integral, queijo branco, sucos e leite desnatado ou semidesnatado) ${ }^{25}$, e menor consumo de carnes, ovos, óleos, gorduras, sementes oleaginosas, açúcares e doces ${ }^{26}$. O fato de o grupo de não consumidores ser constituído apenas por mulheres pode explicar a melhor qualidade da dieta do grupo.

Segundo Carvalho et al. ${ }^{10}$, com dados do ISA-Capital 2003, o consumo de carnes vermelhas e processadas além da recomendação, de acordo os critérios da WCRF ${ }^{1}$, foi relacionado com pior índice da qualidade da dieta em homens. Todos os componentes com análise significativa levam à percepção da melhor qualidade da dieta entre os não consumidores de carnes vermelhas e processadas, uma vez que os indivíduos consumiram mais frutas e leite e menos gordura, álcool e açúcar de adição. Porém, vale ressaltar que a qualidade da dieta não considera somente o consumo de carnes vermelhas e processadas e sim todos os 12 componentes analisados, de modo que comparando os dois grupos, ambos possuem inadequações em diversos parâmetros alimentares, como o consumo de proteínas para acima do recomendado, observado em ambos os grupos, sendo que no caso dos não consumidores pode ser atribuído ao consumo de leguminosas, laticínios e carnes brancas. Por isso, percebe-se que em ambos os grupos a qualidade da dieta em geral pode ser melhorada, independentemente do consumo ou não de carnes vermelhas e processadas. 
Ações que foquem a redução do consumo de carne vermelha e processada, como o projeto "Segunda sem carne", podem contribuir para a redução do consumo excessivo de carne vermelha ${ }^{27}$ e consequente melhoria da qualidade da dieta, com redução da ingestão de proteínas de origem animal e gordura saturada.

Houve também diferença na adequação de ingestão de lipídeos: os consumidores de carnes vermelhas e processadas apresentaram maior percentual de inadequação. Cosgrove et al. ${ }^{22}$, em pesquisa envolvendo a qualidade da dieta de consumidores de carnes vermelhas, brancas e processadas, analisaram que o consumo de gorduras foi significativamente maior $(p<0,001)$ na comparação entre grupos, tanto para homens quanto para mulheres. O consumo excessivo de gorduras, principalmente saturadas, é relatado como possível risco para doenças crônicas não-transmissíveis, destacando-se obesidade central, hipertrigliceridemia e síndrome metabólica ${ }^{5}$. Em pesquisa que analisou o consumo de carne com a qualidade da dieta e mortalidade, o alto consumo de carne processada foi significativamente associado com o aumento da mortalidade por todas as causas ${ }^{23}$.

Sabe-se que no Brasil o consumo de carnes está associado à cultura da população, sendo para a maioria imprescindível sua presença principalmente nas grandes refeições (almoço e jantar). Porém, a parcela de vegetarianos no Brasil vem crescendo principalmente nos últimos anos. Dados do Instituto Brasileiro de Opinião Pública e Estatística ${ }^{28}$ apontam que $8 \%$ dos brasileiros declaram não consumir nenhum tipo de carne. Estes fatos podem ser reflexos dos impactos da carne tanto na saúde (quando consumida em excesso) quanto no meio ambiente, uma vez que a pecuária é responsável por $18 \%$ dos gases de efeito estufa do mundo, ultrapassando a poluição gerada por automóveis ${ }^{29}$. Além disso, dados apontam que a maior parcela dos salários dos brasileiros é destinada à compra de carnes ${ }^{30,31}$, o que corrobora a inflação atual: as carnes são apontadas como um dos principais produtos responsáveis ${ }^{32}$.
Vale ressaltar que não foi encontrado nenhum indivíduo vegetariano nos participantes analisados nesta pesquisa, fato sustentado pela prevalência da alimentação onívora no Brasil, sendo preocupantes os excessos cometidos pela maioria da população, que ultrapassa as recomendações de consumo saudável de carnes, principalmente vermelhas e processadas ${ }^{33}$. A principal limitação deste estudo foi a baixa prevalência de indivíduos não consumidores de carnes vermelhas e processadas, por isso deve-se ter cautela nas extrapolações desses resultados e conclusões. Apesar da pequena amostra, foram encontradas diferenças significativas na qualidade da dieta e ingestão de lipídeos entre os grupos analisados.

Destacam-se, nesta pesquisa, a utilização de amostra probabilística, o cálculo de pontuação para a avaliação da qualidade da dieta, o IQD-R, que ainda é uma ferramenta inovadora para avaliação da qualidade da dieta no Brasil e a estimativa do consumo alimentar habitual por meio de métodos estatísticos inseridos na plataforma do Multiple Source Method.

\section{O N CLUSÃ O}

Sugere-se que no grupo de não consumidores de carne vermelha e processada a qualidade da dieta seja melhor, bem como a adequação da contribuição energética dos lipídeos, em relação ao grupo de consumidores, entretanto inadequações nutricionais foram observadas nos dois grupos.

\section{COLABORADORES}

CFC FREITAS contribuiu na análise e redação do manuscrito. A MENDES, AM CARVALHO, AAF CARIOCA e RM FISBERG colaboraram na análise de dados. DML MARCHIONI colaborou na revisão crítica e análise de dados do manuscrito.

\section{REFERÊ NCIAS}

1. World Cancer Research Fund and American Institute for Cancer Research. Food, nutrition, 
physical activity and the prevention of cancer: A global perspective. London: WCRF; 2007 [2014 jul 5]. Available from: www.dietandcancerreport.org

2. Cross AJ, Leitzmann MF, Gail MH, Hollenbeck AR, Schatzkin A, Sinha R. A prospective study of red and processed meat intake in relation to cancer risk. PLoS Med. 2007; 4(12):e325.

3. Micha R, Wallace SK, Mozaffarian D. Red and processed meat consumption and risk of incident coronary heart disease, stroke, and diabetes: A systematic review and meta-analysis. Circulation. 2010; 121(21):2271-83.

4. Kim E, Coelho D, Blachier F. Review of the association between meat consumption and risk of colorectal cancer. Nutr Res. 2013; 33(12): 983-94.

5. Cocate PG, Natali AJ, de Oliveira A, Alfenas RC, Peluzio MC, Longo GZ, et al. Red but not white meat consumption is associated with metabolic syndrome, insulin resistance and lipid peroxidation in Brazilian middle-aged men. Eur J Prev Cardiol. 2015; 22(2):223-30.

6. Larsson SC, Orsini N. Red meat and processed meat consumption and all-cause mortality: A metaanalysis. Am J Epidemiol. 2014; 179(3):282-9.

7. White DL, Collinson A. Red meat, dietary heme iron, and risk of type 2 diabetes: The involvement of advanced lipoxidation end products. Advances Nutr. 2013; 4(4):403-11.

8. Pham NM, Mizoue T, Tanaka K, Tsuji I, Tamakoshi A, Matsuo K, et al. Meat consumption and colorectal cancer risk: An evaluation based on a systematic review of epidemiologic evidence among the Japanese population. JPN J Clin Oncol. 2014; 44(7):641-50.

9. Zhu H-C, Yang X, Xu L-P, Zhao L-J, Tao G-Z, Zhang $C$, et al. Meat consumption is associated with esophageal cancer risk in a meat-and cancerhistological-type dependent manner. Digest Dis Sci. 2014; 59(3):664-73.

10. Carvalho AM, César CLG, Fisberg RM, Marchioni DML. Excessive meat consumption in Brazil: Diet quality and environmental impacts. Public Health Nutr. 2013; 16(10):1893-9.

11. Instituto Brasileiro de Geografia e Estatística. Pesquisa orçamentos familiares 2008-2009: análise do consumo alimentar pessoal no Brasil. Rio de Janeiro: IBGE; 2011.

12. World Health Organization. Join report of expert consultation on diet, nutrition and the prevention of chronic diseases: Diet, nutrition, and the prevention of chronic diseases. Technical Report Series, n 916. Geneva: WHO; 2002.
13. Previdelli ÁN, de Andrade SC, Pires MM, Ferreira SRG, Fisberg RM, Marchioni DM. Índice de Qualidade da Dieta Revisado para população brasileira. Rev Saúde Pública. 2011; 45(4):794-8.

14. Cesar CLG, Carandina L, Alves MCP, Barros MBA, Goldbaum M. Saúde e condição de vida em São Paulo. Inquérito multicêntrico de saúde no estado de São Paulo - ISA/SP. São Paulo: USP; 2005.

15. Moshfegh AJ, Rhodes DG, Baer DJ, Murayi T, Clemens JC, Rumpler WV, et al. The US Department of Agriculture Automated Multiple-Pass Method reduces bias in the collection of energy intakes. Am J Clin Nutr. 2008; 88(2):324-32.

16. Raper N, Perloff B, Ingwersen L, Steinfeldt L, Anand J. An overview of USDA's dietary intake data system. J Food Comp Anal. 2004; 17(3):545-55.

17. Marchioni DML, Voci SM, Lima FELd, Fisberg RM, Slater B. Reproducibility of a food frequency questionnaire for adolescents. Cad Saúde Pública. 2007; 23(9):2187-96.

18. Fisberg RM, Colucci ACA, Morimoto JM, Marchioni DML. Questionário de freqüência alimentar para adultos com base em estudo populacional. Rev Saúde Pública. 2008; 42(3):550-4.

19. Harttig U, Haubrock J, Knüppel S, Boeing H. The MSM program: Web-based statistics package for estimating usual dietary intake using the Multiple Source Method. Eur J Clin Nutr. 2011; 65:S87-S91.

20. World Health Organization. Physical status: The use of and interpretation of anthropometry. Report of a WHO Expert Committee. Geneva: WHO; 1995.

21. Brasil. Ministério da Saúde. Secretaria de Atenção à Saúde. Guia alimentar para a população brasileira $2^{a}$ ed. Brasília: Ministério da Saúde; 2014.

22. Cosgrove M, Flynn A, Kiely M. Consumption of red meat, white meat and processed meat in Irish adults in relation to dietary quality. Br J Nutr. 2005; 93(6):933-42.

23. Kappeler R, Eichholzer M, Rohrmann S. Meat consumption and diet quality and mortality in NHANES III. Eur J Clin Nutr. 2013; 67(6):598-606.

24. Camilleri GM, Verger EO, Huneau J-F, Carpentier F, Dubuisson C, Mariotti F. Plant and animal protein intakes are differently associated with nutrient adequacy of the diet of French adults. J Nutr. 2013; 143(9):1466-73.

25. Selem SSA, de Castro MA, César CLG, Marchioni DML, Fisberg RM. Associations between Dietary Patterns and Self-Reported Hypertension among Brazilian Adults: A Cross-Sectional Population-Based Study. J Acad Nutr Diet. 2014; 114(8):1216-22.

26. Verly Junior E, Carvalho AM, Fisberg RM, Marchioni DML. Adesao ao guia alimentar para população brasileira. Rev Saúde Pública. 2013; 47(6):1021-7. 
27. Lacerda B, Carvalho A, Martins J, Negrão C, Selem S, Fisberg R, et al. Segunda Sem Carne na Faculdade de Saúde Pública: um projeto de intervenção. Rev Cult Extensão USP. 2013; 10:113-9.

28. Instituto Brasileiro de Opinião Pública e Estatística. Dia mundial do vegetarianismo: $8 \%$ da população brasileira afirma ser adepta ao estilo. 2012 [acesso 2015 fev 2]. Disponível em: http://www.ibope. com.br/pt-br/noticias/paginas/dia-mundial-dovegetarianismo-8-da-populacao-brasileira-afirmaser-adepta-ao-estilo.aspx

29. Schlindwein MM, Kassouf AL. Análise da influência de alguns fatores socioeconômicos e demográficos no consumo domiciliar de carnes no Brasil. Rev Econ Sociol Rural. 2006; 44(3):549-72.

30. Levy-Costa RB, Sichieri R, Pontes NS, Monteiro CA. Disponibilidade domiciliar de alimentos no Brasil: distribuição e evolução (1974-2003). Rev Saúde Pública. 2005; 39(4):530-40.
31. Mondini L, Martins VA, Margarido MA, Bueno CRF, Claro RM, Levy RB. Evolução dos preços de alimentos em São Paulo, Brasil, 1980-2009: considerações sobre o acesso à alimentação saudável. Infor Econ. 2012; 42:47-55.

32. Instituto Brasileiro de Geografia e Estatística. Índice Nacional de Preços ao Consumidor Amplo - IPCA e Índice Nacional de Preços ao Consumidor - INPC. Rio de Janeiro: IPCA; 2014 [acesso 2014 dez 8]. Disponível em: http://www.ibge.gov.br/home/ estatistica/indicadores/precos/inpc_ipca/ipca inpc_201409comentarios.pdf

33. Carvalho AM, Cesar CLG, Fisberg RM, Marchioni DM. Meat consumption in Sao Paulo - Brazil: Trend in the last decade. PLoS One. 2014; 9:e96667.

Recebido: fevereiro 24, 2015 Versão final: abril 24, 2015 Aprovado: maio 28, 2015 
